\title{
ESTUDIO EXPERIMENTAL SOBRE LA REPERCUSIÓN ANATÓMICA DE LA ZÓNULA DE ZINN TRAS LA \\ INTRODUCCIÓN DE UN ANILLO ENDOCAPSULAR EN OJOS DE CERDO
}

\section{ANATOMICAL REPERCUSSION ON THE ZONULAR APPARATUS AFTER A CAPSULAR TENSION RING INSERTION IN SWINE EYES}

\author{
COLOMÉ-CAMPOS J ${ }^{1}$, ORTEGA-USOBIAGA J ${ }^{2}$, SHAHIN $\mathrm{M}^{1}$, FORCADELL $\mathrm{M}^{3}$, PINO A ${ }^{3}$, \\ FORTEZA T ${ }^{3}$, BAUBÍ $\mathrm{C}^{3}$
}

\section{RESUMEN}

Objetivos: Valorar experimentalmente la integridad morfológica de los ligamentos suspensorios de la zónula de Zinn (LSZZ) tras la introducción de un anillo de distensión capsular (ADC) mediante un estudio descriptivo.

Métodos: Se utilizaron siete ojos de cerdo a los cuales se realizó una extracción extracapsular de cristalino transparente. A cuatro se introdujo un $\mathrm{ADC}$ con pinzas y al quinto con inyector. Los otros dos se utilizaron como control. Se examinó la integridad anatómica y la nueva configuración de los LSZZ con un microscopio electrónico de barrido.

Resultados: No se observaron alteraciones en la integridad de los LSZZ. Los LSZZ adoptan tras la introducción de un ADC una nueva trayectoria de repliegue consecuencia de la nueva disposición del saco capsular.

Conclusiones: En un modelo experimental con ojos de cerdo no se encontraron lesiones en los LSZZ por lo que parece que, en cápsulas elásticas y

\section{ABSTRACT}

Purpose: To carry out an experimental and descriptive study that exhibits the anatomical repercussions on the zonular apparatus after a capsular tension ring (CTR) is inserted.

Methods: CTRs were inserted in five swine eyes (four with forceps, one with an injector). Two additional eyes were left untouched for control purposes. The integrity of the suspensory ligament was examined by scanning electron microscopy.

Results: We did not observe alterations in the integrity of the zonular apparatus. The suspensory ligaments adopted a new fold configuration.

Conclusions: CTR insertion is a safe therapeutic tool to maintain the integrity of the lens zonules in elastic, large diameter capsular bags (Arch Soc Esp Oftalmol 2008; 83: 589-594).

Key words: Capsular tension ring, lens zonules, clear lens extraction, zonular dialysis.

\footnotetext{
Recibido: 26/9/07. Aceptado: 25/9/08.

1 Doctor en Medicina. Servicio de Oftalmología. Hospital de Tortosa Verge de la Cinta. Tortosa. España.

2 Doctor en Medicina. Clínica Baviera. Bilbao. España.

${ }^{3}$ Licenciado en Medicina. Servicio de Oftalmología. Hospital de Tortosa Verge de la Cinta. Tortosa. España.
} 
de gran diámetro, la inserción de un ADC no produce roturas en los LSZZ.

Palabras clave: Anillos de distensión capsular, zónula de Zinn, extracción extracapsular de cristalino transparente, diálisis zonular.

\section{INTRODUCCIÓN}

La presencia de defectos en la integridad de los ligamentos suspensorios de la zónula de Zinn (LSZZ) es una circunstancia que dificulta la realización quirúrgica de la catarata mediante facoemulsificación incluso en manos de cirujanos experimentados.

Se conocen diversas maniobras para subsanar este problema quirúrgico, desde la realización de las clásicas y prácticamente obsoletas cirugías intracapsulares o extracapsulares, hasta la introducción o colocación de la lente intraocular en ubicaciones distintas al saco capsular (SC) como la cámara anterior o el sulcus ciliar. Un recurso quirúrgico bastante estandarizado es la introducción de un anillo de distensión capsular (ADC) en el interior del SC.

Es bien conocida, y la experiencia así lo indica desde el inicio de su uso en el año 1991 (1), la efectividad que generan los ADC cuando nos encontramos frente a una debilidad o diálisis de los LSZZ para conservar la integridad anatómica del SC merced a las fuerzas de tracción centrífugas que generan los ADC en ausencia de cristalino. De este modo, es una práctica habitual el colocar los ADC en circunstancias como el síndrome pseudoexfoliativo, miopía magna, aniridia, uveítis y retinosis pigmentaria, además de casos de expresión máxima de debilidad como la diálisis zonular que encontramos en síndromes mal formativos como por ejemplo el de Marfan, Ehlers-Danlos o Weill- Marchesani o bien de etiología postraumática o iatrogénica (2) (3). Hay casos extremos donde estos ADC requieren de un soporte de sujeción que obliga a la necesidad de suturar el mismo en las paredes del globo ocular (4). La técnica de introducción del ADC es manual, bien con la ayuda de unas pinzas o bien con la de un inyector. Sin embargo uno evidencia cuando procede a la colocación del mismo cierta agresión en la integridad de todo el SC y sus ligamentos de estabilización, máxime en la zona directa de inicio de introducción del mismo, que es donde se genera una tracción máxima de tipo tangencial (5). El objetivo de nuestro trabajo es dar a conocer bajo microscopia electrónica y mediante un estudio descriptivo, la magnitud de la posible agresión que genera la utilización de dichos anillos. Si es así, quizás merezca considerar que el uso de los ADC en ciertas circunstancias puede generar unos posibles riesgos que modifiquen la praxis del acto quirúrgico.

\section{SUJETOS, MATERIAL Y MÉTODOS}

Se utilizaron siete ojos de cerdo de ocho meses de edad y provenientes de un matadero municipal que se enuclearon 30 minutos después del sacrificio de los animales. Posteriormente fueron colocados en un recipiente con tampón fosfato salino $0,1 \mathrm{M}$ y conservados a $4^{\circ} \mathrm{C}$. A las 12 horas de la enucleación y tras la escisión del casquete corneo-escleral y el iris se realizó una capsulorrexis de unos $5 \mathrm{~mm}$ de diámetro con la ayuda de un cistotomo y unas pinzas de Utrata procediendo a la extracción extracapsular del cristalino transparente tras maniobras de hidro-expresión. El córtex se aspiró con una cánula tipo Simcoe. Seguidamente se introdujeron en cada ojo una serie de ADC de PMMA que se seleccionaron de forma aleatoria. Los primeros cuatro anillos fueron introducidos con una pinza tipo McPherson y el quinto con la ayuda de un inyector. Para el primer ojo utilizamos un ADC Corneal ${ }^{\circledR}$ modelo ATC110 de $11 \mathrm{~mm}$ de diámetro, para el segundo un ADC Oculaid ${ }^{\circledR}$ modelo PC275 de $12 \mathrm{~mm}$, para el tercer ojo un ADC Morcher ${ }^{\circledR}$ tipo 14 de $12 \mathrm{~mm}$, para el cuarto un ADC Ophtec ${ }^{\circledR}$ de $13 \mathrm{~mm}$ y para el quinto introducimos un ADC AMO Injector Ring ${ }^{\circledR}$ de $11 \mathrm{~mm}$ (tabla I). Como control utilizamos dos ojos, uno al que únicamente se había realizado una extracción extracapsular de cristalino transparente pero sin la introducción de anillo y otro al que se había conservado el cristalino. Para acabar procedimos a resecar la pared posterior del globo ocular con la finalidad de obtener una perspectiva anterior y posterior de los LSZZ. 
Tabla I. Anillos de distensión capsular utilizados en el presente estudio

\begin{tabular}{lll}
\hline Anillos de distensión capsular & Material & Diámetro \\
\hline Corneal $^{\circledR}$ ATC 110 & PMMA & $11 \mathrm{~mm}$ \\
Oculaid $^{\circledR}$ PC275 & PMMA & $12 \mathrm{~mm}$ \\
Morcher $^{\circledR} 14$ & PMMA & $12 \mathrm{~mm}$ \\
Ophtec $^{\circledR}$ & PMMA & $13 \mathrm{~mm}$ \\
AMO $^{\circledR}$ Injector Ring & PMMA & $11 \mathrm{~mm}$ \\
\hline \hline
\end{tabular}

Los globos oculares se introdujeron en una sustancia fijadora de glutaraldehido al $6 \%$ según la técnica de Karnovsky (6) y se conservaron a $4^{\circ} \mathrm{C}$ un mínimo de 2 horas hasta su procesamiento.

Realizamos lavados de las muestras con tampón fosfato y se fijaron con tetraosmio de óxido para proceder a su deshidratación a través de una batería de alcoholes de concentraciones crecientes y acabar en una solución de aniloacetato. Tras obtener el punto crítico con $\mathrm{CO} 2$ se recubrieron las muestras con oro por el procedimiento de «sputtering». La distancia de trabajo utilizada fue de $39 \mathrm{~mm}$ y el microscopio electrónico de barrido utilizado fue un JSM-6400 propiedad de la Universidad Rovira y Virgili.

Se seleccionaron los LSZZ de los cuadrantes de los cuatro meridianos principales de cada ojo en pasos de 130 micrómetros y se observó su integridad, medida en número de roturas fibrilares, y la densidad, contabilizando el número de fibras dentro de los 130 micrómetros considerados. También se estudió la configuración del trayecto de los LSZZ observando si mantenían su disposición rectilínea habitual, y la inserción a nivel del saco capsular, registrando el número de desinserciones. El estudio se realizó desde una perspectiva anterior comparándose con los ojos control y se registraron las imágenes a aumentos de 10X, 75X, 150X, 200X, 250X, 300X, 400X, 600X, 1000X y 2500X.

\section{RESULTADOS}

No se detectaron alteraciones en la integridad de los LSZZ en ninguno de los ojos estudiados, puesto que en ninguno de los cuadrantes valorados, tanto para los que se introdujo un ADC, bien con inyector (fig. 1) o no (figs. 2, 3 y 5), como para los ojos control (fig. 4), se observaron roturas ni tampoco desinserciones. La densidad se mantuvo estable con unas cifras medias de 43 fibras por cada 130 micró-

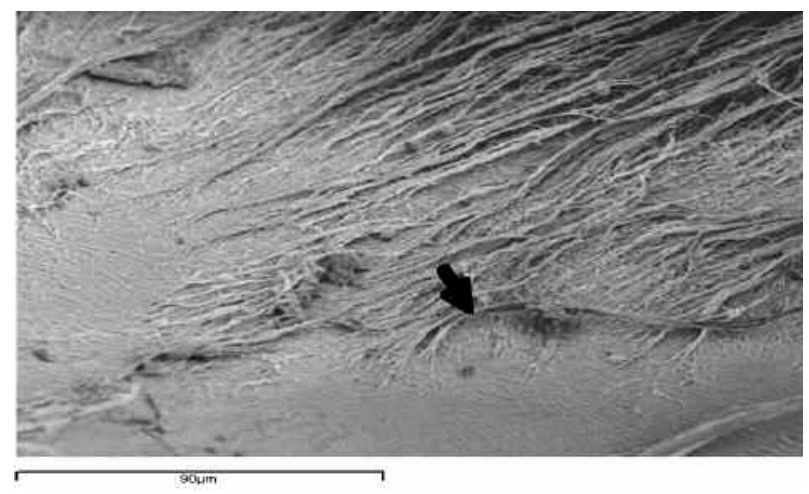

Fig. 1: Imagen de microscopia electrónica de barrido (x600) donde se muestra la integridad de los ligamentos suspensorios en su inserción al SC (flecha) después de la introducción de un ADC con inyector.

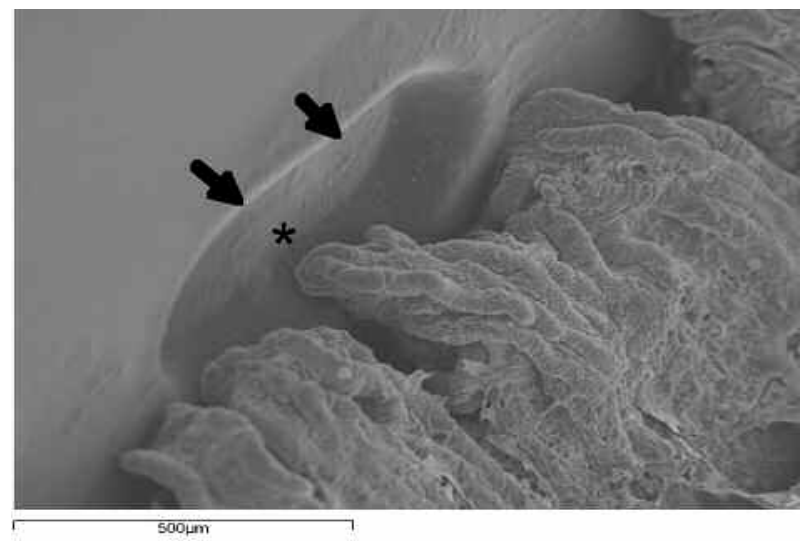

Fig. 2: Imagen de microscopía electrónica de barrido (x150) que muestra la impronta que genera el SC (*) al adaptarse a los pequeños orificios que figuran en el presente ADC (flecha).

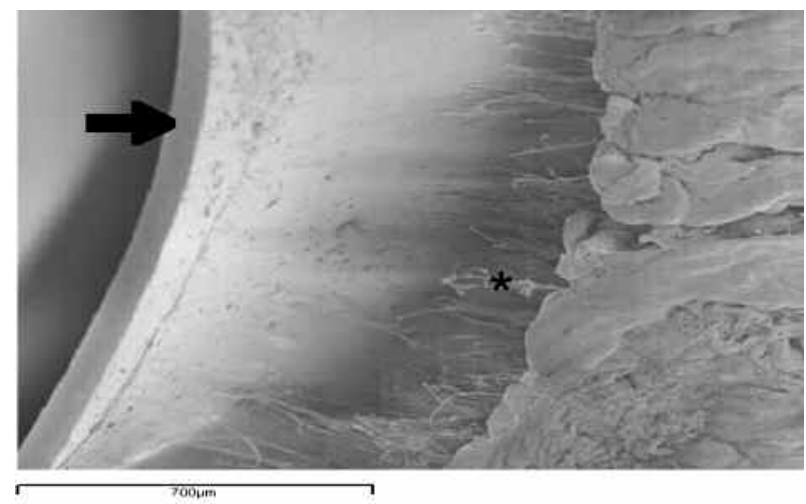

Fig. 3: Imagen de microscopía electrónica de barrido (x75) que muestra el relieve de los bordes de la capsulorrexis (flecha) y de la zónula de Zinn (*). 


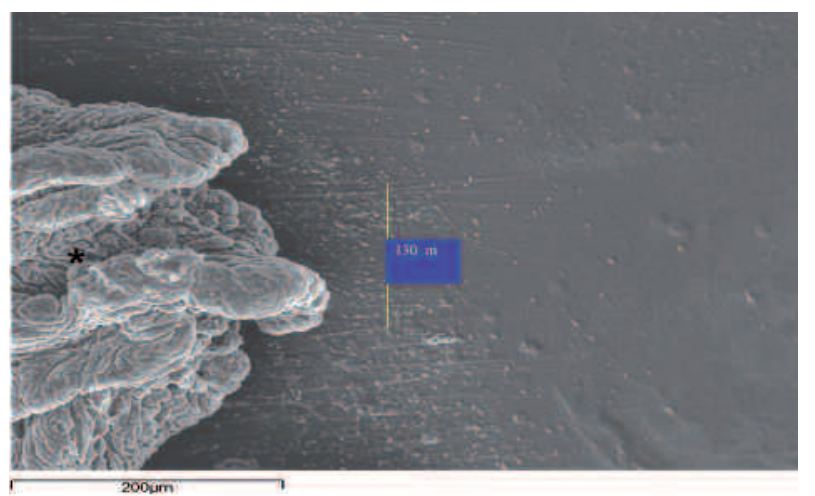

Fig. 4: Imagen de microscopia electrónica de barrido (x100) que muestra el músculo ciliar (*) y densidad de las fibras para uno de los cuadrantes estudiados en un ojo control.

metros, muy semejante a la de los ojos control que fue de 46 fibras.

Sí se encontraron alteraciones en la configuración o trayecto de los LSZZ comparando con los ojos control, puesto que la presencia de un saco capsular en ausencia de cristalino pero con la introducción de un ADC hizo que se objetivaran cambios en la arquitectura de la red tridimensional de los LSZZ, de manera que estos pierden la configuración rectilínea habitual presentando una nueva trayectoria y disposición en forma de repliegue y angulación posterior (fig. 6).

\section{DISCUSIÓN}

El estudio de presente trabajo se ha realizado sobre ojos de cerdo de corta edad. Es de suponer que la integridad de todo el sistema suspensorio del cristalino así como la elasticidad del SC se encuentran perfectamente conservados (7). Al extrapolar el diseño del trabajo en humanos cabría considerar que el ADC se utiliza normalmente en ojos que presentan zónulas supuestamente debilitadas, donde la resistencia a la tracción en el transcurso de la cirugía de la catarata es mucho menor que en circunstancias normales, tal como se ha podido observar en tinciones oculares realizadas postmortem (8). Por ejemplo se ha demostrado que en los síndromes pseudoexfoliativos la resistencia de los LSZZ a la tracción es cuatro veces menor, e incluso en algunos pacientes las fibras se encuentran rotas o deterioradas antes de realizar el acto quirúrgico (9).

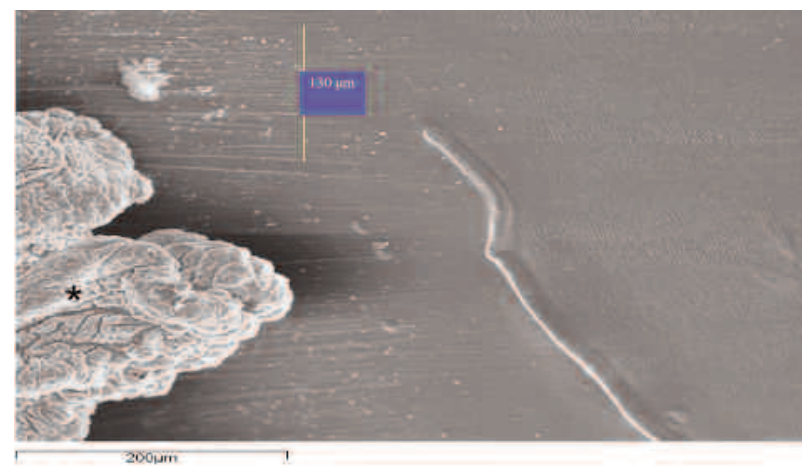

Fig. 5: Imagen de microscopia electrónica de barrido (x100) que muestra el músculo ciliar (*) y la densidad de la fibras en su inserción al SC para uno de los cuadrantes estudiados en un ojo con ADC. No se objetivan cambios anatómicos respecto al ojo control.

El SC de los ojos de cerdo se estima de un diámetro superior al del humano (10), a pesar de ello en nuestro estudio, al tratarse de ojos jóvenes hemos objetivado un $\mathrm{SC}$ de un diámetro medio de 12,1 mm (fig. 7), ligeramente mayor al SC del ojo humano que es de 10,3 a 10,8 mm de diámetro cuando se encuentra vacío $(11,12)$. Nosotros hemos utilizado ADC diseñados para su uso en humanos y de un tamaño medio de $11 \mathrm{~mm}$. Podemos imaginar que la introducción de un ADC en un SC de mayor tamaño requiere una menor fricción-resistencia que la introducción en un SC de tamaño menor.

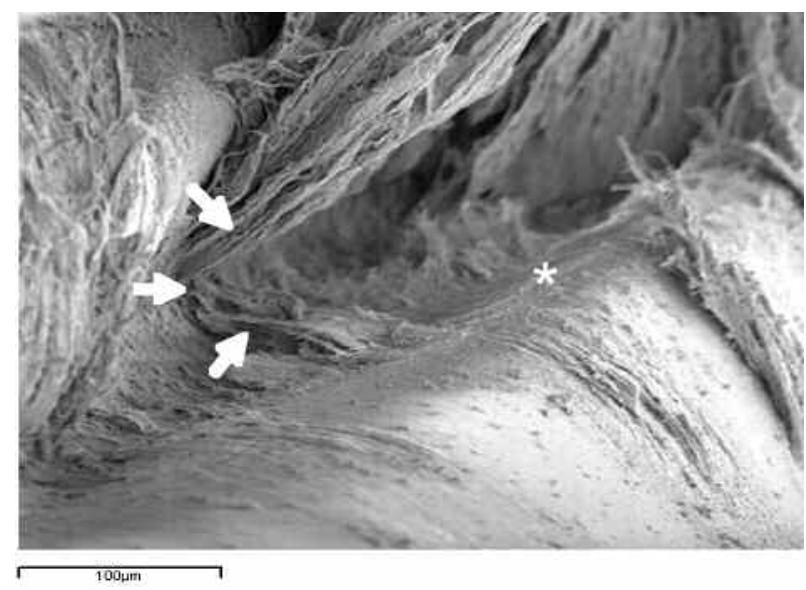

Fig. 6: Imagen de microscopia electrónica de barrido (x300) que muestra el dorso del ecuador del SC del cristalino (*) tras la introducción de un ADC y la nueva configuración de repliegue posterior que adoptan los ligamentos suspensorios (flecha). 


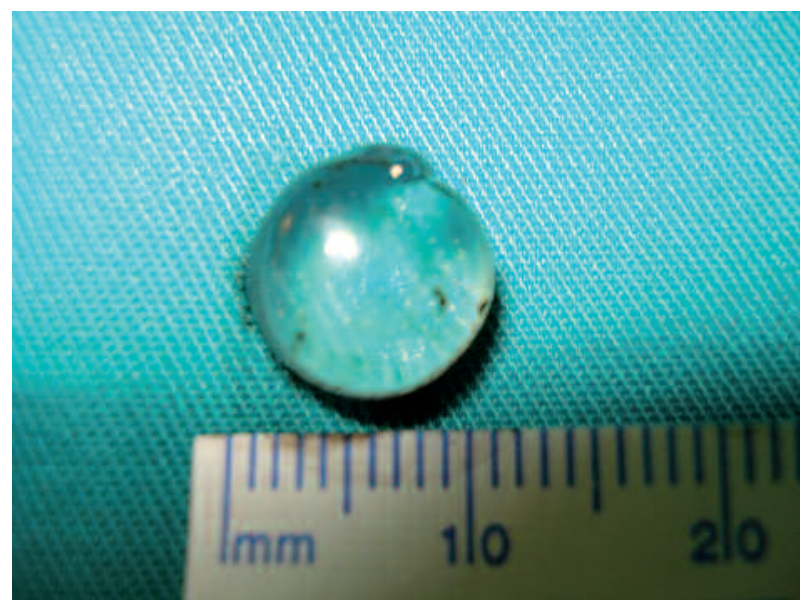

Fig. 7: Diámetro del cristalino de uno de los ojos de cerdo utilizados.

La maniobra de introducción de un ADC es relativamente fácil en condiciones normales, sin embargo en algunos casos complejos o si la entrada se realiza de una forma demasiado traumática o incluso si el diseño del ADC no es el más adecuado puede generar severas tracciones sobre el «fórnix» del SC que pueden llegar incluso hasta perforarlo (2). Se ha descrito que la utilización de ADC con la ayuda de inyectores puede evitar o minimizar las fuerzas de tracción sobre los LSZZ y el SC, es con este fin que hemos utilizado uno de los ojos para objetivar la repuesta microscópica tras su introducción.

Los LSZZ presentan una enorme capacidad distensiva y una importante firmeza en sus inserciones capsulares y ciliares (13). Se ha descrito que la fibras pueden aumentar su longitud hasta cuatro veces la original $(14,15)$ sin evidenciar hallazgos de rotura, fisuras ni desinserciones. Sin embargo con el transcurso de los años los LSZZ se hacen más finos y decrecen en número condicionando una variabilidad importante interpersonal de resistencia traccional (16), circunstancias a considerar cuando realizamos maniobras que puedan alterar la integridad anatómica previamente debilitada.

Hemos encontrado alteraciones en la trayectoria de los LSZZ comparando con los ojos control, y suponemos que son debidas a que los ADC generan unas fuerzas centrífugas sobre el ecuador del SC que provocan cierta distensión sobre los LSZZ. También habría que contemplar que con la extracción del cristalino queda reducida la biconvexidad del SC, factor a considerar para interpretar la nueva disposición de los LSZZ y su posible repercusión en el mecanismo de la acomodación.

\section{BIBLIOGRAFÍA}

1. Hara T, Hara T, Yamada Y. «Equator ring» for maintenance of the completely circular contour of the capsular bag equator after cataract removal. Ophthalmic Surg 1991; 22: 358-359.

2. Menapace R, Findl O, Georgopoulos M, Rainer G, Vass $C$, Schmetterer $K$. The capsular tension ring: designs, applications, and techniques. J Cataract Refract Surg 2000; 26: 898-912.

3. Pitrová S. A distance ring for stabilization of the lens capsule (a multicenter clinical study). Cesk Slov Oftalmol 1998; 54: 299-304.

4. Tokuda Y. In the bag IOL suture fixation represents a new approach to zonular dehiscence. Ocular Surgery News 1997; 8: 9.

5. Colomé Campos J, Pérez Moreda F, Belmonte Martínez J. Estudio experimental de la acción de los anillos endocapsulares en relación a la extensión de la diálisis zonular. Microcirugia Ocular 1999; 7: 33-38.

6. Karnovsky MJ. A formaldehyde-glutaraldehyde fixative of high osmolality for use in electron microscopy. J Cell Biol 1965; 27: 137-138.

7. Krag S, Olsen T, Andreassen TT. Biomechanical characteristics of the human anterior lens capsule in relation to age. Invest Ophthalmol Visc Sci 1997; 38: 357-363.

8. Wilson DJ, Jaeger MJ, Green WR. Effects of extracapsular cataract extraction on the lens zonules. Ophthalmology 1987; 94: 467-470.

9. Guzek JP, Holm M, Cotter J, Cameron JA, Rademaker WJ, Wissinger $\mathrm{DH}$, et al. Risk factors for intraoperative complications in 1000 extracapsular cataract cases. Ophthalmology 1987; 94: 461-466.

10. Lee DH, Lee HY, Lee KH, Chung KH, Joo CK. Effect of a capsular tension ring on the shape of the capsular bag and opening and the intraocular lens. J Cataract Refract Surg 2001; 27: 452-456.

11. Galand A, Bonhomme L, Collée M. Direct measurement of the capsular bag. J Am Intraocul Implant Soc 1984; 10: 475-476.

12. Richburg FA, Sun HS. Size of the crushed cataractous capsule bag. J Am Intraocul Implant Soc 1983; 9: 333335.

13. Streeten BW, Licari PA. The zonules and the elastic microfibrillar system in the ciliary body. Invest Ophthalmol Vis Sci 1983; 24: 667-681.

14. Raviola G. The fine structure of the ciliary zonule and ciliary epithelium. With special regard to the organization an insertion of the zonular fibrils. Invest Ophthalmol 1971; 10: 851-869.

15. Canals M, Potau JM, Costa J. Estructura y propiedades elásticas de la zónula cristaliniana. Arch Soc Esp Oftalmol 1999; 74: 131-136.

16. Buschmann W, Linnert D, Hofmann W, Gross A. The tensile strength of human zonule and its alteration with age. Albrecht Von Graefes Arch Klin Exp Ophthalmol 1978; 206: 183-190. 